\title{
Relationship of Bladder Function with Toe Position Sense, Neurological Level, Lesion Severity, and Bulbocavernosus Reflex in Patients with Spinal Cord Injury
}

\author{
Fazıl KULAKLI', Murat ERSÖZ1', Pınar EGÜZ1', Kurtuluş KÖKLÜ'1, Sumru ÖZEL'1, Gülten KARACA², Canan ÇULHA \\ ${ }^{\prime}$ Clinic of Physical Medicine and Rehabilitation, Ankara Physical Medicine and Rehabilitation Training and Research Hospital, Ankara, Turkey \\ ${ }^{2}$ Clinic of Physical Medicine and Rehabilitation, Konya Training and Research Hospital, Konya, Turkey
}

\begin{abstract}
Objective: Examination of the relationship among toe position sense (TPS), neurological level (cervical, thoracic, lumbosacral), severity of the lesion (complete, incomplete), bulbocavernosus reflex (BCR), and bladder behavior in patients with spinal cord injury (SCI).

Material and Methods: Fifty-nine patients with SCI were included in the study. Patient characteristics, TPS, and BCR were recorded. Neurological level and lesion severity were determined according to the American Spinal Injury Association Impairment Scale (AIS) classification. Maximum cystometric capacity (MC), storage and emptying function, type of detrusor (overactive, acontractile-underactive), spontaneous voided urine volume (SVU), and post-void residual urine volume (PVR) values were established performing the urodynamic study.

Results: Neurological level, lesion severity, TPS, and BCR were not significantly correlated with storage dysfunction, emptying dysfunction, type of detrusor, MC, and PVR ( $p>0.05)$. SVU was significantly lower in patients without TPS than in patients with TPS and in complete lesions than in incomplete lesions $(p<0.05)$.

Conclusion: The presence of TPS could be a predictive finding in terms of SVU. We can say that neurological level, lesion severity, and BCR are not adequate parameters to predict bladder dysfunction; therefore, urodynamic evaluation is necessary for each patient with SCI.

Keywords: Spinal cord injury, bladder function, deep sensation
\end{abstract}

\section{Introduction}

Spinal cord micturition center is primarily localized at the S2-4 level, generally located at the L1 vertebral level (1). After the period of spinal shock, complete suprasacral injuries result in detrusor hyperreflexia and detrusor sphincter dyssynergia (DSD) $(1,2)$ and complete sacral injuries result in detrusor areflexia (3). However, some studies identified no definite correlation between somatic neurologic findings and characteristic urodynamic findings $(4,5)$.
Lower urinary tract (LUT) dysfunction is a prominent cause of morbidity and mortality in spinal cord injury $(\mathrm{SCl})$ patients $(6,7)$. Protecting upper urinary system function, preventing urinary tract infection, providing low bladder pressure, and obtaining full emptying ability are aimed at by evaluating patients neuro-urologically. Urodynamic studies are the gold standard for evaluating sphincter and bladder function in patients with $\mathrm{SCl}(8,9)$.

The medial lemniscus-dorsal column pathway is an ascending spinal tract, carrying deep sensory information to the brain;

Address for Correspondence: Fazıl Kulaklı, MD, Ankara Fizik Tedavi ve Rehabilitasyon Eğitim ve Araştırma Hastanesi, Fiziksel Tıp ve Rehabilitasyon Kliniği, Ankara, Türkiye. Phone: +90505 3121016 E-mail: drfzl46@gmail.com

Received: August 2013 Accepted: August 2014

(C)opyright 2015 by Turkish Society of Physical Medicine and Rehabilitation - Available online at www.ftrdergisi.com

Kulaklı F, Ersöz M, Egüz P, Köklü K, Özel S, Karaca G, Çulha C. Relationship of Toe Position Sense, Neurological Level, Lesion Severity and Bulbocavernosus Reflex with Bladder Function in Patients with Spinal Cord Injury 
however, it is not evaluated in the American Spinal Injury Association Impairment Scale (AIS). Many studies have investigated the relationship between AIS and bladder function, but only one has investigated the relationship between deep sensation and bladder function. In this study, toe position sense (TPS) was examined as deep sensation in $\mathrm{SCl}$ patients, and at the end of the 1 -year follow-up evaluation, the frequency of volitional voiding in patients with protected TPS in the first 72 hours after SCl was higher than that in patients without TPS; the authors indicated that this finding may be predictive of volitional voiding (10).

The main objective of the present study was to investigate the relationship between TPS and bladder function in patients with $\mathrm{SCl}$.

\section{Material and Methods}

Fifty-nine patients (46 males, 13 females) in the rehabilitation program in our hospital with the diagnosis of $\mathrm{SCl}$ were evaluated prospectively. Patients with the following characteristics were not included in the study: present spinal shock period; pervious urodynamic study; previous LUT dysfunction; neurogenic bladder due to other causes (diabetes mellitus, previous stroke or brain injury, other neurologic diseases etc.); $\mathrm{SCl}$ out of lesion level; brain injury and pelvic trauma accompanying $\mathrm{SCl}$; and previous use of pharmacological agents, such as anticholinergics, alpha blockers, baclofen, and injections, for urinary bladder. The study protocol was approved by the ethical committee of our institution.

Patient age, etiological factor, presence of TPS, and bulbocavernosus reflex (BCR) were recorded. TPS and BCR were evaluated by the same doctor making physical examination and determined as present or absent. Neurological level and severity of the lesion were set according to AIS. Maximum cystometric capacity (MC), storage and emptying function, type of detrusor, spontaneous voided urine volume (SVU), and post-void residual urine volume (PVR) values were assessed by urodynamic studies. For urodynamic testing, a computer-assisted urodynamic unit (Libra+ MMS, Enschede, The Netherlands) was used. The patients were positioned in supine position. The bladder was filled, and the intravesical pressure was recorded via a sterile transurethral double-lumen 8-F catheter. Sterile saline solution at body temperature was used as a filling medium, and the bladder was filled at a rate of $50 \mathrm{~mL} / \mathrm{min}$. MC was defined as a patient experienced sensation that would normally lead to immediate evacuation of the bladder, the volume at the time as intravesical pressure was above $40 \mathrm{~cm} \mathrm{H}_{2} \mathrm{O}$, and the volume at the time as the beginning of voiding without control (11). Storage dysfunction was defined as $M C$ was less than $300 \mathrm{~mL}$ and emptying dysfunction was defined as PVR was $50 \mathrm{~mL}$ or more (12). Detrusor overactivity (DO) was defined as a spontaneously non-volutional detrusor contractions in the storage phase of urodynamic study (11).

\section{Statistical Analysis}

Statistical analysis was performed using Statistical Package for the Social Sciences (SPSS Inc., Chicago, IL, USA) 15.0, and the level of statistical significance was defined as $p<0.05$. De- scriptive statistics, Chi-square tests, Mann-Whitney U test, and Kruskal-Wallis test were used.

\section{Results}

Etiological factor, neurological level, lesion severity, presence of TPS and BCR, frequency of storage and emptying dysfunction, and detrusor type of patients are shown in Table 1. Mean age was established as $36.8 \pm 16.3$ years, median value of post-injury duration was 232 days $(\min =35$; $\max =2190)$, median MC was $243 \mathrm{~mL}(\mathrm{~min}=37$; $\max =750$ ), median PVR was $280 \mathrm{~mL}(\mathrm{~min}=0$; $\max =750$ ), and median $\mathrm{SVU}$ was $0 \mathrm{~mL}(\mathrm{~min}=0$; $\max =600$ ). According to TPS, neurological level, lesion severity, and BCR; emptying dysfunction, storage dysfunction, and type of detrusor rates are shown in Table 2. There was no significant relationship between TPS and emptying dysfunction, storage dysfunction, type of detrusor, and PVR values ( $p>0.05$; Table 2; Table 3). SVU was significantly higher in patients with TPS than in those without TPS $(p<0.05$; Table 3$)$. According to neurological level, emptying dysfunction, storage dysfunction, PVR, and SVU values were not significantly different ( $p>0.05$; Table 2; Table 3). Although the frequency of DO was higher and detrusor acontractility-underactivity was lower in patients with cervical injury than in patients with thoracic and lumbosacral injuries, there was no significant difference between neurological level and type of detrusor ( $p>0.05$; Table 2). No significant difference was found between lesion severity and emptying dysfunction, storage dysfunction, type of detrusor, and PVR values ( $p>0.05$; Table 2; Table 3). However, SVU was significantly lower in complete lesions than in incomplete lesions $(p<0,05$; Table 3$)$. There was no significant difference between $B C R$ and emptying dysfunction, storage dysfunction, type of detrusor, SVU, and PVR values ( $p>0.05$; Table 2; Table 3). According to presence of TPS, neurological level, lesion severity and presence of BCR; median (min-max) MC values are shown in Table 3. According to TPS, neurological level, lesion severity, and $B C R$, median $M C$ values were not significantly significant $(p>0.05)$.

Table 1. Variance of patients in terms of etiological factor, neurological level, lesion severity, presence of TPS and BCR, frequency of storage, and emptying dysfunction and type of detrusor

\begin{tabular}{lc} 
& $\mathbf{n = 5 9}$ \\
\hline Etiological factor (Traumatic/Non-traumatic) & $54 / 5$ \\
Neurological level (Cervical/Thoracic/Lumbosacral) & $13 / 30 / 16$ \\
Lesion severity (Complete/Incomplete) & $34 / 25$ \\
TPS (Present/Absent) & $20 / 39$ \\
BCR (Present/Absent) & $50 / 9$ \\
Frequency of storage dysfunction & 35 \\
Frequency of emptying dysfunction & 56 \\
Type of detrusor (Overactive/Acontractile-underactive) & $48 / 11$ \\
\hline TPS: Toe position sense; BCR: bulbocavernosus reflex &
\end{tabular}


Table 2. According to presence of TPS, neurological level, lesion severity, and presence of BCR, rates of emptying dysfunction, storage dysfunction, and type of detrusor

\begin{tabular}{|c|c|c|c|c|}
\hline & \multicolumn{2}{|c|}{ Type of dysfunction } & \multicolumn{2}{|c|}{ Type of detrusor } \\
\hline & $\begin{array}{c}\text { Emptying } \\
\text { dysfunction }\end{array}$ & $\begin{array}{c}\text { Storage } \\
\text { dysfunction }\end{array}$ & Overactive & $\begin{array}{l}\text { Acontractile- } \\
\text { underactive }\end{array}$ \\
\hline & n (\%) & n (\%) & n (\%) & n (\%) \\
\hline \multicolumn{5}{|l|}{ TPS } \\
\hline TPS present & $18(90 \%)$ & $11(55 \%)$ & $17(85.0 \%)$ & $3(15.0 \%)$ \\
\hline TPS absent & $38(97.4 \%)$ & $24(61.5 \%)$ & $31(79.5 \%)$ & $8(20.5 \%)$ \\
\hline$\chi^{2} ; p$ values & $1.515 ; 0.218$ & $0.234 ; 0.628$ & \multicolumn{2}{|c|}{$0.265 ; 0.607$} \\
\hline \multicolumn{5}{|c|}{ Neurological level } \\
\hline Cervical & $11(84.6 \%)$ & $8(61.5 \%)$ & $12(92 \%)$ & $1(8 \%)$ \\
\hline Thoracic & $30(100 \%)$ & $18(60 \%)$ & $24(80 \%)$ & $6(20 \%)$ \\
\hline Lumbosacral & 15 (93.8\%) & $9(56 \%)$ & $12(75 \%)$ & $4(25 \%)$ \\
\hline$\chi^{2} ; p$ values & $4.510 ; 0.105$ & $0.095 ; 0.954$ & \multicolumn{2}{|c|}{$1.490 ; 0.475$} \\
\hline \multicolumn{5}{|c|}{ Lesion severity } \\
\hline Complete & $33(97 \%)$ & $21(61 \%)$ & 27 (79\%) & $7(21 \%)$ \\
\hline Incomplete & $23(92 \%)$ & $14(56 \%)$ & $21(84 \%)$ & $4(16 \%)$ \\
\hline$\chi^{2} ; p$ values & $0.764 ; 0.382$ & $0.198 ; 0.656$ & \multicolumn{2}{|c|}{$0.200 ; 0.655$} \\
\hline \multicolumn{5}{|l|}{ BCR } \\
\hline BCR present & $47(94 \%)$ & $30(60 \%)$ & $42(84.0 \%)$ & $8(16.0 \%)$ \\
\hline BCR absent & $9(100 \%)$ & $5(55.5 \%)$ & $6(66.6 \%)$ & $3(33.3 \%)$ \\
\hline$\chi^{2} ; p$ values & $0.569 ; 0.451$ & $0.062 ; 0.803$ & \multicolumn{2}{|c|}{$1.511 ; 0.219$} \\
\hline
\end{tabular}

TPS: Toe position sense; BCR: bulbocavernosus reflex

Table 3. Comparison of TPS, neurological level, lesion severity, and BCR with median (min-max) values of SVU, PVR, and MC

\begin{tabular}{|c|c|c|c|c|c|c|}
\hline & SVU & $p$ values & PVR & $p$ values & MC (Mean \pm SD) & $p$ values \\
\hline \multicolumn{7}{|l|}{ TPS } \\
\hline TPS present & $140(0-600)$ & \multirow{2}{*}{0.000} & $195(0-750)$ & \multirow{2}{*}{0.109} & $246.5(37-620) \mathrm{mL}$ & \multirow{2}{*}{0.873} \\
\hline TPS absent & $0(0-300)$ & & $300(0-750)$ & & $243(67-750) \mathrm{mL}$ & \\
\hline \multicolumn{7}{|l|}{ Neurological level } \\
\hline Cervical & $0(0-400)$ & \multirow{3}{*}{0.101} & $190(0-650)$ & \multirow{3}{*}{0.118} & $165(37-620)$ & \multirow{3}{*}{0.226} \\
\hline Thoracic & $0(0-300)$ & & $370(0-750)$ & & $244(67-750)$ & \\
\hline Lumbosacral & $110(0-600)$ & & $202.5(0-550)$ & & $296.5(143-570)$ & \\
\hline \multicolumn{7}{|l|}{ Lesion severity } \\
\hline \multirow[t]{2}{*}{ Complete Incomplete } & $0(0-300)$ & \multirow{2}{*}{0.002} & $300(0-750)$ & \multirow{2}{*}{0.213} & $246.5(79-750)$ & \multirow{2}{*}{0.854} \\
\hline & $100(0-600)$ & & $200(0-750)$ & & $220(37-620)$ & \\
\hline \multicolumn{7}{|l|}{ BCR } \\
\hline BCR present & $0(0-600)$ & \multirow{2}{*}{0.355} & $290(0-750)$ & \multirow{2}{*}{0.941} & $232(37-750) \mathrm{mL}$ & \multirow{2}{*}{0.562} \\
\hline BCR absent & $0(0-120)$ & & $270(125-500)$ & & $279(83-608)$ & \\
\hline
\end{tabular}

TPS: Toe position sense; MC: maximum cystometric capacity; BCR: bulbocavernosus reflex; PVR: post-void residual urine volume; SVU: spontaneous voided urine volume; SD: standard deviation

\section{Discussion}

In this study, the main aim was to evaluate the relationship between TPS and bladder function. In addition, relationships of neurological level, lesion severity, and BCR with bladder behav- ior were investigated. According to TPS, neurological level, lesion severity, and $B C R$, storage dysfunction, emptying dysfunction, and PVR were not significantly different. The frequency of DO was higher and detrusor acontractility-underactivity was 
lower in patients with TPS than in those without TPS. However, according to TPS, neurological level, lesion severity, and BCR, type of detrusor and median MC values were not significantly different. SVU was significantly higher in patients with TPS and incomplete lesions than in patients without TPS and complete lesions. However; there was no significant difference between BCR and SVU.

Deep sensations (position, vibration, proprioception) and $B C R$ are not evaluated in AIS classification. BCR is a sacral polysynaptic reflex. Evaluating BCR gives an idea about sacral somatic S2-4 nerves and also gives an indirect indication of bladder function. If BCR disappears, bladder dysfunction due to lower motor neuron lesion could occur; but urodynamic character of detrusor muscle could not be determined by examining BCR alone (13). Weiss et al. (10) reported that the frequency of volitional voiding in patients with protected TPS in the first 72 hours after $\mathrm{SCl}$ was more than that in patients without TPS, and this finding might be predictive of volitional voiding. Shenot et al. (14) showed that pinprick sensation and presence of BCR might be predictive of volitional voiding, but they were not sensitive about predicting detrusor hyperreflexia and sphincter dyssynergia. According to Wyndaele (15), presence of BCR and anal reflex were significantly associated with $\mathrm{DO}$ and sphincter dyssynergia and absence of BCR was significantly associated with detrusor acontractility. In our study, TPS was examined as deep sensation and presence of BCR was evaluated. We detected that TPS and BCR were not associated with storage dysfunction, emptying dysfunction, type of detrusor, and MC values. However, we established that SVU in patients with TPS was significantly higher than in patients without TPS, similar to results of Weiss et al. (10) There was no significant relationship between BCR and SVU. The presence of TPS may be a predictive finding in terms of SVU, but further studies are needed to evaluate the association between deep sensation, BCR, and bladder function.

After the period of spinal shock, complete suprasacral injuries result in DO and DSD $(1,2)$ and complete sacral injuries usually result in detrusor acontractility (3). However, some studies reported that neurological findings were not always associated with urodynamic findings in suprasacral and sacral injuries $(4,5)$; there was no significant differences among the patients with cervical, thoracic, and lumbar injuries in terms of $M C(16,17)$ and DSD (18) and also LUT dysfunction could be determined in patients with normal neurological findings by performing urodynamic studies $(19,20)$. In our study, neurological level was not associated with type of detrusor, storage dysfunction, emptying dysfunction, MC, SVU, and PVR.

Studies investigating lesion severity and bladder function found no significant difference between complete and incomplete lesions in terms of maximum detrusor pressure and $\mathrm{MC}$ (21); mean MC and mean intravesical leak-point pressures were not significantly different between AIS-A and AIS B-E patients (22); although DO was more benign in AIS-B patients than in AIS-A patients, neuropathic bladder type and compliance were not significantly different between AIS-A and AIS-B patients (23). Scivoletto et al. (24) have shown that none of the patients with AIS-A impairment on admission reached volitional void- ing at 5 months; AIS-B patients had a $90 \%$ lower probability of achieving good bladder control and AIS-C patients had $65 \%$ lower than AIS-D patients. In our study, lesion severity was not significantly associated with storage dysfunction, emptying dysfunction, type of detrusor, MC, and PVR. However, similar to Scivoletto's study, SVU values of complete lesions were significantly lower than in incomplete lesions.

Relationship of neurological level and lesion severity with bladder behavior in patients with $\mathrm{SCl}$ were investigated in different studies and different opinions were reported. In our study, we detected that according to neurological level and lesion severity, storage dysfunction, emptying dysfunction, type of detrusor, MC, and PVR values were not significantly different, but a significant relationship in terms of SVU was established. Based on supporting data from the literature, we can say that determining neurological level and lesion severity may give an opinion about bladder function; SVU can be lower in patients without TPS and complete lesions than in patients with TPS and incomplete lesions. However, bladder function could not be determined clearly, optimum treatment could not be arranged, and neurogenic bladder complications could not be prevented by evaluating these findings alone. In addition to clinical assessment, all patients with $\mathrm{SCl}$ should be evaluated by urodynamic studies to appraise neurogenic bladder.

\section{Study Limitations}

The bladder was filled at a rate of $50 \mathrm{~mL} / \mathrm{min}$ in our study. Although this filling rate is the upper limit of medium fill cystometry, it may provoke detrusor overactivity in $\mathrm{SCl}$ patients with overactive detrusors. Choosing the individualized filling rate (body weight-kg/4) would be more suitable.

Absence of data regarding DSD appeared as a limitation of our study. Although we performed sphincter EMG in the majority of our patients, it could not be performed in all patients because of some technical problems (EMG electrode, EMG cable problems, etc.). Further studies in which sphincter EMG is performed uniformly will help determination of the contribution of DSD to emptying dysfunction.

In addition, TPS was evaluated by physical examination and was determined according to patients' subjective answers, so the results cannot be accepted as fully objective findings.

\section{Conclusion}

The presence of TPS can be a predictive finding for SVU in patients with $\mathrm{SCl}$. However, further studies are needed to determine the relationship between deep sensation and bladder function. Neurological level, lesion severity, and BCR are not adequate parameters to predict bladder dysfunction, so urodynamic evaluation is necessary for each patient.

Ethics Committee Approval: The study was approved by the ethics committee of Ankara Physical Medicine and Rehabilitation Training and Research Hospital.

Informed Consent: All patients were informed about the study and informed consent was signed by all of the patients. 
Peer-review: Externally peer-reviewed.

Author Contributions: Concept - F.K.; Design - F.K., K.K.; Supervision F.K., M.E., S.Ö., C.Ç., G.K.; Resource - F.K., P.E.; Materials - F.K., P.E.; Data Collection and/or Processing - F.K., P.E.; Analysis and/or Interpretation F.K., M.E., K.K.; Literature Review - F.K., P.E.; Writer - F.K.; Critical Review - S.Ö., C.Ç., G.K.

Conflict of Interest: No conflict of interest was declared by the authors.

Financial Disclosure: The authors declared that this study has received no financial support.

\section{References}

1. Erol B, Koçak T, Kadıoğlu A, Müslümanoğlu L, Karamehmetoğlu Ş, Akıncı $M$, et al. The relationship between level of injury and bladder behavior in patients with post-traumatic spinal cord injury. Ulus Travma Acil Cerrahi Derg 2009;15:377-82.

2. Thomas DG, O'Flynn KJ. Spinal cord injury. In: Mundy TP, Stephenson TP, Wein AJ, ed. Urodynamics: Principles, Practice, Application. 1994; London, Churchill Livingstone. p. 345.

3. Fam B, Yalla SV. Vesicourethral dysfunction in spinal cord injury and its management. Semin Neurol 1988;8:150-5. [CrossRef]

4. Blaivas JG, Sinha HP, Zayed AA, Labib KB. Detrusor-external sphincter dyssynergia. J Urol 1981;125:542-4.

5. Blaivas JG. The neurophysiology of micturition. A clinical study of 550 patients. J Urol 1982;127:958-63.

6. Stöhrer M, Blok B, Castro-Diaz D, Chartier-Kastler E, Del Popolo G, Kramer $G$, et al. EAU guidelines on neurogenic lower urinary tract dysfunction. Eur Urol 2009;56:81-8. [CrossRef]

7. Schäfer W, Abrams P, Liao L, Mattiasson A, Pesce F, Spangberg A, et al. Good urodynamic practices: uroflowmetry, filling cystometry, and pressure-flow studies. Neurourol Urodyn 2002;21:261-74. [CrossRef]

8. Watanabe T, Rivas DA, Chancellor MB. Urodynamics of spinal cord injury. Urol Clin North Am 1996;23:459-73. [CrossRef]

9. Linsenmeyer TA, Culkin D. APS recommendations for the urological evaluation of patients with spinal cord injury. J Spinal Cord Med 1999;22:139-42.

10. Weiss DJ, Fried GW, Chancellor MB, Herbison GJ, Ditunno JF Jr, Staas WE Jr. Spinal cord injury and bladder recovery. Arch Phys Med Rehabil 1996;77:1133-5. [CrossRef]

11. Abrams P, Cardozo L, Fall M, Griffiths D, Rosier P, Ulmsten U. The standardisation sub-committee of the international continence society. The standardisation of terminology in lower urinary tract function:
Report from the standardisation sub-committee of the international continence society. Urology 2003;61:37-49. [CrossRef]

12. Ersoz M, Tunc $H$, Akyuz M, Ozel S. Bladder storage and emptying disorder frequencies in hemorrhagic and ischemic stroke patients with bladder dysfunction. Cerebrovasc Dis 2005;20:395-9. [CrossRef]

13. Akyuz M, Selçuk B. Omurilik yaralanmasında elektrofizyolojik tetkiklerin prognozdaki yeri. Turk J Phys Med Rehab 2005;48:6.

14. Shenot PJ, Rivas DA, Watanabe T, Chancellor MB. Early predictors of bladder recovery and urodynamics after spinal cord injury. Neurourol Urodyn 1998;17:25-9. [CrossRef]

15. Wyndaele JJ. Correlation between clinical neurological data and urodynamic function in spinal cord injured patients. Spinal Cord 1997;35:213-6. [CrossRef]

16. Yildiz N, Alkan H, Akkaya N, Catalbas N, Ardic F. Relationship of lesion level and severity with bladder behavior in patients with spinal cord injury. Turk J Phys Med Rehab 2011;57:206-11.

17. Pannek J, Greving I, Tegenthoff M, Nediat S, Bötel U, May B, et al. Urodynamic and rectomanometric findings in patients with spinal cord injury. Neurourol Urodyn 2001;20:95-103. [CrossRef]

18. Schurch B, Schmid DM, Karsenty G, Reitz A. Can neurologic examination predict type of detrusor sphincter-dyssynergia in patients with spinal cord injury? Urology 2005;65:243-6. [CrossRef]

19. Watanabe T, Vaccaro AR, Kumon H, Welch WC, Rivas DA, Chancellor MB. High incidence of occult neurogenic bladder dysfunction in neurologically intact patients with thoracolumbar spinal injuries. J Urol 1998;159:965-8. [CrossRef]

20. Patki P, Woodhouse J, Hamid R, Shah J, Craggs M. Lower urinary tract dysfunction in ambulatory patients with incomplete spinal cord injury. J Urol 2006;175:1784-7. [CrossRef]

21. Akkoc Y, Cinar Y, Kismali E. Should complete and incomplete spinal cord injury patients receive the same attention in urodynamic evaluations and ultrasonography examinations of the upper urinary tract? Int J Rehabil Res 2012;35:178-80. [CrossRef]

22. Moslavac S, Dzidic I, Kejla Z. Neurogenic detrusor overactivity: comparison between complete and incomplete spinal cord injury patients. Neurourol Urodyn 2008;27:504-6. [CrossRef]

23. Rapidi C, Petropoulou K, Galata A, Fragkaki M, Kandylakis E, Venieri $M$, et al. Neuropathic bladder dysfunction in patients with motor complete and sensory incomplete spinal cord lesion. Spinal Cord 2008;46:673-8. [CrossRef]

24. Scivoletto G, Cosentino E, Morganti B, Farchi S, Molinari M. Clinical prognostic factors for bladder function recovery of patients with spinal cord and cauda equina lesions. Disabil Rehabil 2008;30:330-7. [CrossRef] 